\title{
Till stress do us ataRT: a novel toxin-antitoxin system targeting translation initiation
}

\author{
Mariavittoria Pizzinga ${ }^{1}$, Robert F Harvey ${ }^{1}$ and Anne E Willis ${ }^{\star, 1}$
}

Cell Death and Differentiation (2017) 24, 951-952; doi:10.1038/cdd.2017.66; published online 12 May 2017

Protein synthesis is a highly regulated, three-stage process, comprised of initiation, elongation and termination. In brief, during initiation, the messenger RNA (mRNA) binds to the mRNA channel on the small ribosomal subunit and the startcodon is positioned within the P-(peptidyl) decoding site. A charged initiator transfer RNA (tRNA), which in prokaryotes is normally (formyl) methionyl-tRNAi (met-tRNA ${ }^{\text {fMet }}$ ) and in eukaryotes is tRNA ${ }^{i M e t}$, is recruited to the start-codon. Importantly, by decoding the first triplet of the mRNA, the initiator tRNA determines the reading frame. The large ribosomal subunit joins to form a ribosomal complex with the initiator tRNA in the P-site of the ribosome, which is primed for the elongation stage, where the message is decoded. During the elongation process, an aminoacyl-tRNA that has an anticodon complementary to the mRNA codon is recruited to the ribosomal A-site. A peptide bond is formed by transfer of the amino acid/peptide attached to the tRNA in the P-site to the aminoacyl-tRNA in the adjacent A-site, and the newly formed peptidyl-tRNA is subsequently translocated from the A-site to the $\mathrm{P}$-site, in conjunction with the mRNA. These processes are promoted by both the ribosome and elongation factors. When a termination codon enters the A-site, termination factors bind to the ribosome and promote the hydrolysis of the peptidyl-tRNA. ${ }^{1}$

The process of mRNA translation is highly regulated via altering the bioavailability and/or function of its components. ${ }^{1}$ Although tRNAs were previously considered to have a fairly "passive" role in the overall regulation of protein synthesis, the recent data suggest that, through their modification and bioavailability, they make a major contribution to the global regulation of mRNA translation in all domains of life. With hindsight, this is perhaps unsurprising, since tRNAs play a central role in protein synthesis by providing the link between mRNAs and amino acids. Amino acids are attached to tRNAs through aminoacylation, which is catalysed by aminoacyltRNA synthetases. The high level of precision with which the processes of aminoacylation and decoding occur is, in part, achieved through post-transcriptional modification of tRNAs, with over 100 different post-transcriptional modifications described. $^{2}$ Many of the modifications required to maintain accuracy during decoding target position 34 , the wobble nucleotide, and position 37, the nucleotide 3'-adjacent to the anticodon. ${ }^{3}$ Some modifications, such as methylation resulting in N1-methylguanosine at position 37 ( $\left.\mathrm{m}^{1} \mathrm{G} 37\right)$, are conserved amongst all three domains of life. ${ }^{4}$ Interestingly, while the majority of tRNA modifications are constitutive, some are dependent on cell state and are influenced by growth or exposure to stress. For example, the tRNA modification enzyme glucose-inhibited division protein (GidA), which catalyses the addition of a carboxymethylaminomethyl group to position 5 of the anticodon wobble uridine in conjunction with the GTPase $\mathrm{MnmE}^{5}$ is important for the survival of Streptococcus mutans following cell stress. ${ }^{6}$

In eukaryotic cells, the binding of tRNA ${ }^{\text {iMet }}$ is highly regulated and dependent upon cell state. Accordingly, by modulating tRNA ${ }^{\text {iMet }}$ availability, overall control of initiation, and therefore, of mRNA translation, can be achieved. ${ }^{1}$ In general, this occurs through phosphorylation of the alpha subunit of eukaryotic initiation factor 2 (elF2), which results in an inhibition of Met-tRNA $A_{i}$ binding to ribosomes (see refs 7,8 for reviews). In prokaryotes, control of initiation through regulation of tRNA ${ }^{f M e t}$ bioavailability has been less well described. However, it is known that in addition to the types of alterations of RNA moiety of the aminoacylated tRNA described above, it is also possible to modify the aminoacyl group. In the April issue of Nature Chemical Biology, Jurenas and colleagues (Jurenas et al. 2017) use an elegant combination of classic biochemistry approaches to unveil a novel bacterial type II toxin-antitoxin complex, AtaT-AtaR, that acetylates the free amino group of the aminoacyl moiety of Met-tRNA $^{\text {fMet }}$ and controls protein synthesis (Figure 1).

Proteins of the Gcn5-related N-acetyltransferase (GNAT) family have recently been described as taking part in TA modules in other bacteria. ${ }^{9}$ Therefore, the observation that AtaT contained a GNAT domain lead to the initial hypothesis that the open reading frames for the AtaT and AtaR, located very close to each other in the genome, could represent a previously undescribed TA locus. Generally, translation is a favourite target of class II TA toxins. ${ }^{10}$ In Jurenas et al., the authors show that translation is very strongly inhibited by AtaT in presence of its substrate acetyl-coA, resulting in cell growth arrest. ${ }^{11}$ AtaT is found to specifically target Met-tRNA ${ }^{\text {fMet }}$ for acetylation at the free amino group of the aminoacyl moiety. That a single acetylation would have such a decisive impact on translation is not surprising, as the aminoacyl moiety is critical

\footnotetext{
${ }^{1}$ Department of Toxicology, Medical Research Council Toxicology Unit, Lancaster Road, Leicester LE1 9HN UK

*Corresponding author: A Willis, Department of Toxicology, Medical Research Council Toxicology Unit, Lancaster Road, Leicester, LE1 9HN UK. Tel: +44 (0)116-252 5544; E-mail: aew5@leicester.ac.uk
} 


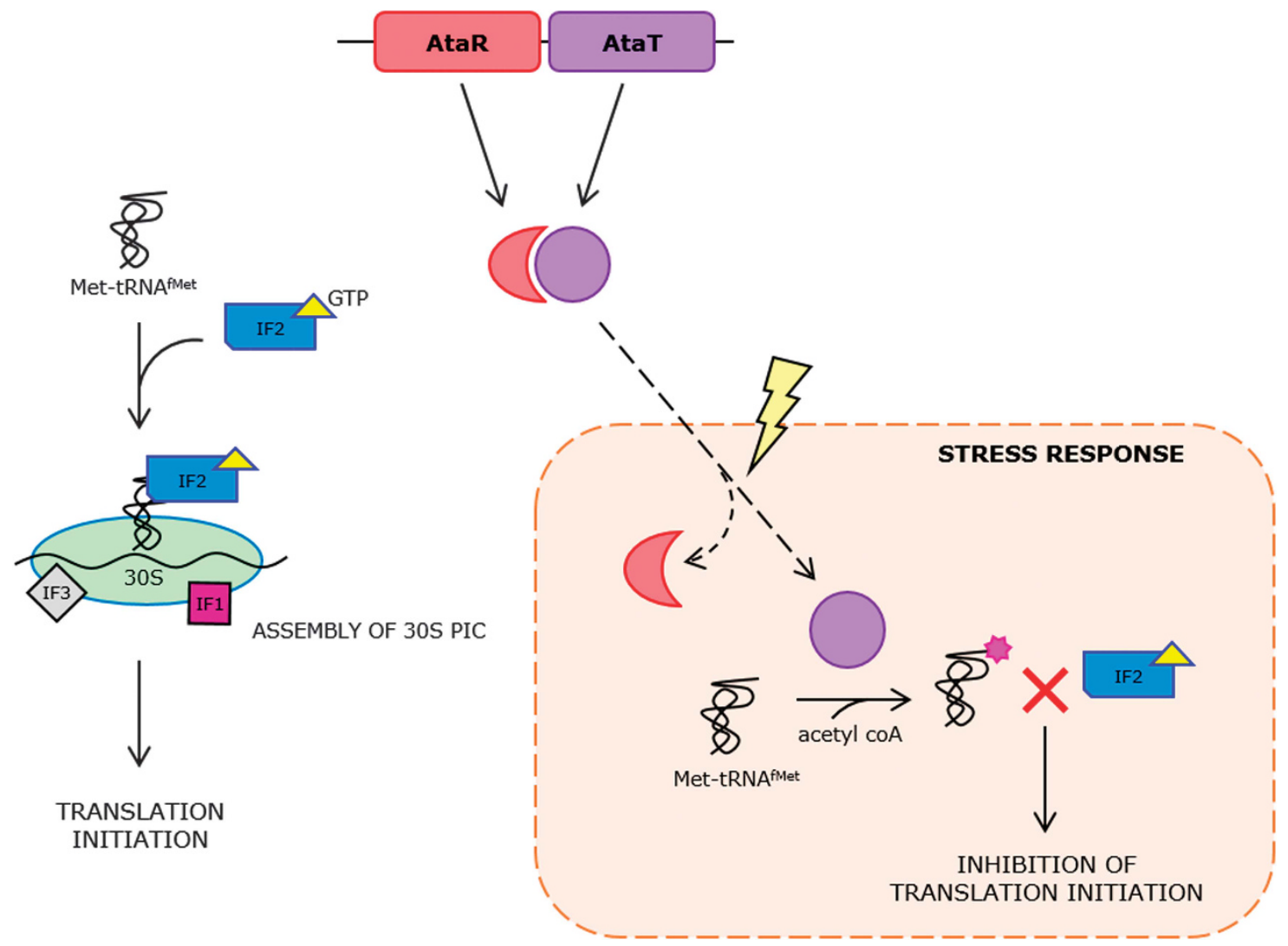

Figure 1 Summary of the ataRT system. In normal conditions, the AtaT toxin is found in a complex with its antitoxin AtaR, which inhibits its acetyltransferase activity. When AtaT is released from this complex following a stress stimulus, it acetylates Met-tRNAfMet using acetyl coA as a substrate. The acetylation precludes the interaction between IF2 and Met-tRNAfMet, resulting in the inhibition of translation initiation

in the interaction of the tRNA with translation initiation factor IF2. The resulting conformational change, therefore, interferes with such interaction, preventing assembly of the initiation complex. As expected, the effects of AtaT are counteracted by expression of its anti-toxin, AtaR, which can prevent acetylation of Met-tRNA, but not reverse it.

The specificity of AtaT to a single tRNA, which allows for selective arrest of translation initiation, distinguishes the AtaTAtaR module from the acetyltransferase toxin TacT described in Salmonella, which instead targets several tRNA species, thus resulting in a block of translation at the elongation level. ${ }^{9}$ The parallel that authors draw with other TA families, the members of which diverge from each other for target specificity and molecular mechanisms, hints at a system evolved to be as versatile as possible. Such flexibility might also be mirrored in the variable stoichiometries observed for the AtaT/AtaR complex, which might represent another level of fine-tuning of the system. ${ }^{11}$

The impact of the AtaT/AtaR module on the ability of $E$. coli to respond to environmental adversities remains to be explored. However, the TacT parallel in Salmonella suggests that it could aid bacteria transit to a drug-tolerant persistent state. Moreover, the observation that AtaR can prevent but not reverse acetylation and the fact that acetylated Met-tRNA ${ }^{\mathrm{fMet}}$ is a dead-end product leave one wondering about the possible mechanism of growth resumption upon withdrawal of the stress stimulus. As has been shown to be the case in the TacT system, it is plausible that detoxifying enzymes might be in place. A better understanding of such mechanisms could have invaluable implications for treatment of infections that respond poorly to current antibiotics.

Importantly, even in eukaryotes, the degree of posttranscriptional modification of tRNAs is unparalleled among all other RNA species, both for abundance and chemical diversity. ${ }^{12}$ The biological function of several of these modifications has often eluded scientists. The findings of this paper could therefore prove to be paradigm-changing, by helping to unravel the emerging role that tRNA modifications play in the control of translation in eukaryotes, especially within organelles such as mitochondria, both in physiological conditions and in disease.

\section{Conflict of Interest}

The authors declare no conflict of interest.

\footnotetext{
1. Hershey JW et al. Cold Spring Harb Perspect Biol 2012; 4: 1-10.

2. Machnicka MA et al. RNA Biol 2014; 11: 1619-1629.

3. Goto-lto $S$ et al. Biomolecules 2017; 7: 32.

4. Marbaniang CN, Vogel J. Curr Opin Microbiol 2016; 30: 50-57.

5. Fislage $\mathrm{M}$ et al. Biopolymers 2016; 105: 568-579.

6. Li D et al. Appl Environ Microbiol 2014; 80: 97-103.

7. Hinnebusch AG, Lorsch JR. Cold Spring Harb Perspect Biol 2012; 4: 1-25.

8. Pavitt GD, Ron D. Cold Spring Harb Perspect Biol 2012; 4: 1-13.

9. Cheverton AM et al. Mol Cell 2016; 63: 86-96.

10. Goeders N, Van Melderen L. Toxins (Basel) 2014; 6: 304-324.

11. Jurenas $D$ et al. Nat Chem Bio 2017 (e-pub ahead of print)

12. Kirchner S, Ignatova Z. Nat Rev Genet 2015; 16: 98-112.
} 